\title{
Effect of Aging on the Physical, Chemical and Dielectric Properties of Dodecylbenzene
}

\author{
I. L. Hosier, J. E. A. Koilraj and A. S. Vaughan \\ Tony Davis High Voltage Laboratory \\ University of Southampton \\ Southampton, SO17 1BJ, UK
}

\begin{abstract}
High voltage transformers and cables form integral parts of the electrical power distribution network and therefore their reliable operation is crucial to preventing costly outages. A liquid dielectric, typically mineral oil, is used in transformers whilst dodecylbenzene is gradually replacing mineral oil in paper/oil cable systems. As such, the oil serves as a convenient medium for sampling to assess plant health. In the current investigation, samples of dodecylbenzene cable oil were aged at $135{ }^{\circ} \mathrm{C}$ under conditions where the oxygen diffusion into the oil was deliberately limited. The resulting aged samples were subjected to a battery of tests to determine changes in their physical, chemical and dielectric properties. Aging resulted in yellowing, oxidation, increased water content, increased dielectric loss, increased electrical conductivity and reduced electrical breakdown strength. Copper accelerated the aging process resulting in the formation of an insoluble precipitate; however, the inclusion of this material did not affect the electrical properties of the oil indicating that it is non-polar in nature. Instead, the deterioration of the dielectric properties was found to be correlated with the production of small polar molecular compounds, which remain dissolved within the oil, such as water, alcohols, ketones, carboxylic acids and aldehydes.
\end{abstract}

Index Terms - Dodecylbenzene, aging, oxidation, dielectric breakdown strength.

\section{INTRODUCTION}

INSULATING oils are widely using in high voltage plant such as cables and transformers and as such provide a convenient medium for sampling to provide an indication of plant health. With much of the equipment in the UK now approaching the end of its expected serviceable lifetime [1, 2], routine condition monitoring is essential to minimize outages and maximize remaining asset lifetime. In recent years considerable interest has been placed on the usage of environmental friendly biodegradable oils in the power industry in order to reduce its overall impact on the environment [3-5]. However, due to its widespread use, early laboratory studies focused on the aging behavior of mineral oil [6-14] and various physical, chemical and dielectric changes were reported following aging. Physical markers of aging include yellowing [6, 7], precipitate formation [7, 8] and changes in oil viscosity [9]. Chemical markers include oxidation $[7,10,11]$, increased acidity $[7,8,9,12,13]$, increased water content $[7,12,13]$ and production of gases and chemical byproducts $[10,14]$. Finally increases in dielectric loss $[7,9,12]$ and a reduction in electrical breakdown strength also accompany aging $[8,9,15]$. In addition the catalytic effects of copper on oil aging are well appreciated $[7,11,14,16]$.

Manuscript received on 18 April 2016, in final form 29 July 2016, accepted 29 July 2016.
Commercial dodecylbenzene cable oil (DDB) is a mixture of isomers with a molecular weight similar to $\mathrm{C}_{18} \mathrm{H}_{30}$. It has excellent biodegradability in the environment $[16,17]$ and is therefore gradually replacing mineral oil in paper/oil cable systems [18] whereas in transformers it is more cost effective to utilize a vegetable oil [3]. The aging behavior of DDB has been previously studied [19-22] and the aging chemistry has been elucidated in the simpler system of single isomer 1phenyldodecane [21]. More sustainable replacements for mineral oils in high voltage plant are vegetable oils and silicone oils and the aging behavior of each has also been studied [23, 24]. Comparison of the general markers of aging in mineral oil, vegetable oil, silicone oil and DDB points to a number of "universal" indicators which could be used for condition monitoring, these are; coloration, oxidation, increased acidity, increased water content and increased dielectric loss. However these earlier studies did not consider the effects of aging on dielectric properties at high fields more appropriate for high voltage plant. When this was done previously in silicone and vegetable oils $[25,26]$ anomalous results were obtained; i.e. the electrical breakdown strength increased with aging time. This was thought to be due to an increase of viscosity in the aged oils which brings into question the value of electrical breakdown measurements as a reliable indicator of aging.

In the current investigation, DDB was again aged at high 
temperatures (i.e. under accelerated laboratory conditions) but the focus was on elucidating the effects of aging on high field phenomena (electrical conductivity and breakdown strength) to determine whether such measurements provide reliable indicators of aging where the viscosity does not increase after aging [23]. To better approximate conditions in plant, large volumes of oil were aged in covered tall glass vials to restrict oxygen diffusion into the oil. Whilst still not ideal, this approach allows the effects of reduced oxygen availability (oxygen which might for example, arise due to degradation of cellulose) to be studied whilst maintaining the same aging temperature used in previous laboratory aging studies. DDB, like mineral oil, is also prone to precipitate formation, particularly when aged in the presence of copper [18, 20-22] and therefore the electrical properties of the particulate was also studied. UV/Vis spectroscopy was used to determine the extent of aging, infrared spectroscopy (IR) and measurements of water content were used to identify chemical changes whilst dielectric loss measurements were complemented here by electrical breakdown and conductivity measurements.

\section{EXPERIMENTAL}

The same commercial dodecylbenzene cable oil (BICC type C148 batch 5808) was used as in previous investigations [18, $20,22]$. In order to restrict oxygen diffusion into the oils, separate large volume $(150 \mathrm{ml})$ oil samples were aged in tall glass jars which were each covered with a Petrie dish. Separate samples were aged for 72, 168, 240 and $336 \mathrm{~h}$ which represent convenient whole days of aging uniformly spaced throughout the total aging time. The aging temperature of $135^{\circ} \mathrm{C}$ was provided by a fan oven whilst copper, in the form of $0.1 \mathrm{~mm}$ thickness sheets, was inserted into selected samples prior to aging so as to provide a total surface area of $96 \mathrm{~cm}^{2}$. The copper sheets were scrubbed thoroughly with sand paper to remove the oxide layer and then washed with acetone before being placed immediately into the oil. Separate samples were then removed from the oven after the prescribed aging time had elapsed. The copper concentration, aging temperature and times were chosen to ensure consistency with earlier studies [7, 18, 20-23]. Some oils were tested after centrifuging (4000 RPM, $10 \mathrm{~min}$ ) to remove the precipitate, whereas others were homogenized to include this material.

Ultraviolet/visible (UV/Vis) spectroscopy was performed using a Perkin Elmer Lambda 35 spectrometer. $10 \mathrm{ml}$ PMMA cuvettes were employed with a path length of $10 \mathrm{~mm}$ and scans were performed from 300 to $1100 \mathrm{~nm}$. Infrared (IR) spectroscopy was performed using a Perkin Elmer spectrum GX instrument. Samples were injected into a test cell composed of opposing $\mathrm{KBr}$ windows with a $0.1 \mathrm{~mm}$ polyethylene gasket and spectra were collected over the range $4000-600 \mathrm{~cm}^{-1}(2,500-16,700 \mathrm{~nm})$ The water content of $1 \mathrm{ml}$ samples was determined using an Aquamax KF titrator (GR Scientific) and at least 3 repeat tests were performed.

Measurements of capacitance and dielectric loss were performed using a Solartron 1296 dielectric interface linked to a Schlumberger SI 1260 impedance-gain-phase analyzer. The test cell was composed of a $35 \mathrm{~mm}$ diameter cup and a $30 \mathrm{~mm}$ diameter plate. Oil was placed in the cup, the plate was then lowered on top and a spacing of $0.2 \mathrm{~mm}$ was maintained using a PTFE slug ( 2 mm square). Measurements were made at room temperature from $0.1 \mathrm{~Hz}$ to $100 \mathrm{kHz}$ using an applied voltage of $1 \mathrm{~V}_{\mathrm{rms}}$ and an integration time of 10 cycles. Relative permittivity values were calculated by dividing the measured capacitance values by that of the empty test cell (i.e. with air as dielectric). Electrical conductivity measurements were performed using a concentric cylindrical test cell of $5 \mathrm{ml}$ capacity with a gap of $2 \mathrm{~mm}$. A voltage was applied to the test cell using a computer controlled system employing a Spellman SL10 high voltage DC supply and a Keithley 6487 picoammeter was used to measure the resulting current. The voltage was increased in $100 \mathrm{~V}$ increments to $4 \mathrm{kV}$ (2 $\mathrm{kV} / \mathrm{mm}$ ), recording the current $10 \mathrm{~s}$ after application of the voltage to allow capacitive charging currents to decay. The electrical conductivity was calculated using the equation;

$$
\sigma=\frac{I L}{V A}
$$

where $I$ is the measured current, $L$ is the gap between the electrodes, $V$ is the applied voltage and $A$ is the electrode area. AC breakdown measurements were performed using a system built in house incorporating a TTi TG330 function generator, power amplifier, high voltage transformer and a current trip. The test cell ( $8 \mathrm{ml}$ volume) is a Perspex cylinder containing opposing $25 \mathrm{~mm}$ diameter chrome steel ball bearings with a separation of $1 \mathrm{~mm}$. The applied AC $(50 \mathrm{~Hz})$ voltage was increased at $350 \mathrm{~V} / \mathrm{s}$ and the voltage at breakdown was then noted. The oil was changed after each test and the ball bearings after every 5 tests to reduce the effects of pitting. Tests were performed after allowing the oil to settle for $\sim 2$ mins, to eliminate air bubbles.

\section{RESULTS}

\subsection{UV/VIS SPECTROSCOPY}

Figure 1 contains spectra from homogenized samples aged without copper. The absorption edge shifts to longer

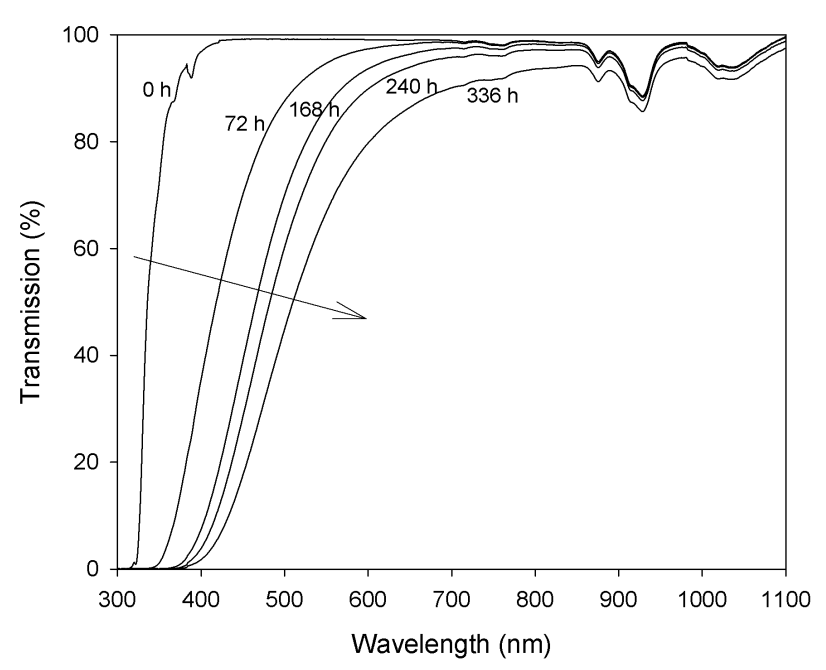

Figure 1. UV/Vis spectra from samples aged without copper. 
wavelengths (arrowed) and the oil becomes visibly yellowed $[6,7,18,20-23]$; the wavelength of $50 \%$ transmission after $336 \mathrm{~h}$ aging is $500 \mathrm{~nm}$, which agrees with previous comparable studies $[18,20]$. In homogenized oils aged with copper (Figure 2a), aging is accelerated and an additional absorbance is present centered at $680 \mathrm{~nm}$ after $72 \mathrm{~h}$ aging (arrowed). This has previously been associated with the presence of copper carboxylates, an aging product that is unique to DDB [18, 20, 21]. Further aging then results in absorption over all wavelengths as a precipitate is formed [7, 8, 18, 20-22]. However, compared to identically aged small volume samples of DDB cable oil [18, 20] the rate of precipitate generation is reduced (i.e. reduced absorption is evident here after any given aging time).

Figure $2 \mathrm{~b}$ shows a plot of absorbance against aging time for two different wavelengths which provides a measure, in homogenized samples, of the concentration of the precipitate. After a $\sim 72 \mathrm{~h}$ induction time the absorbance increases approximately linearly with aging time, indicating a more or less constant rate of precipitate production after $168 \mathrm{~h}$.
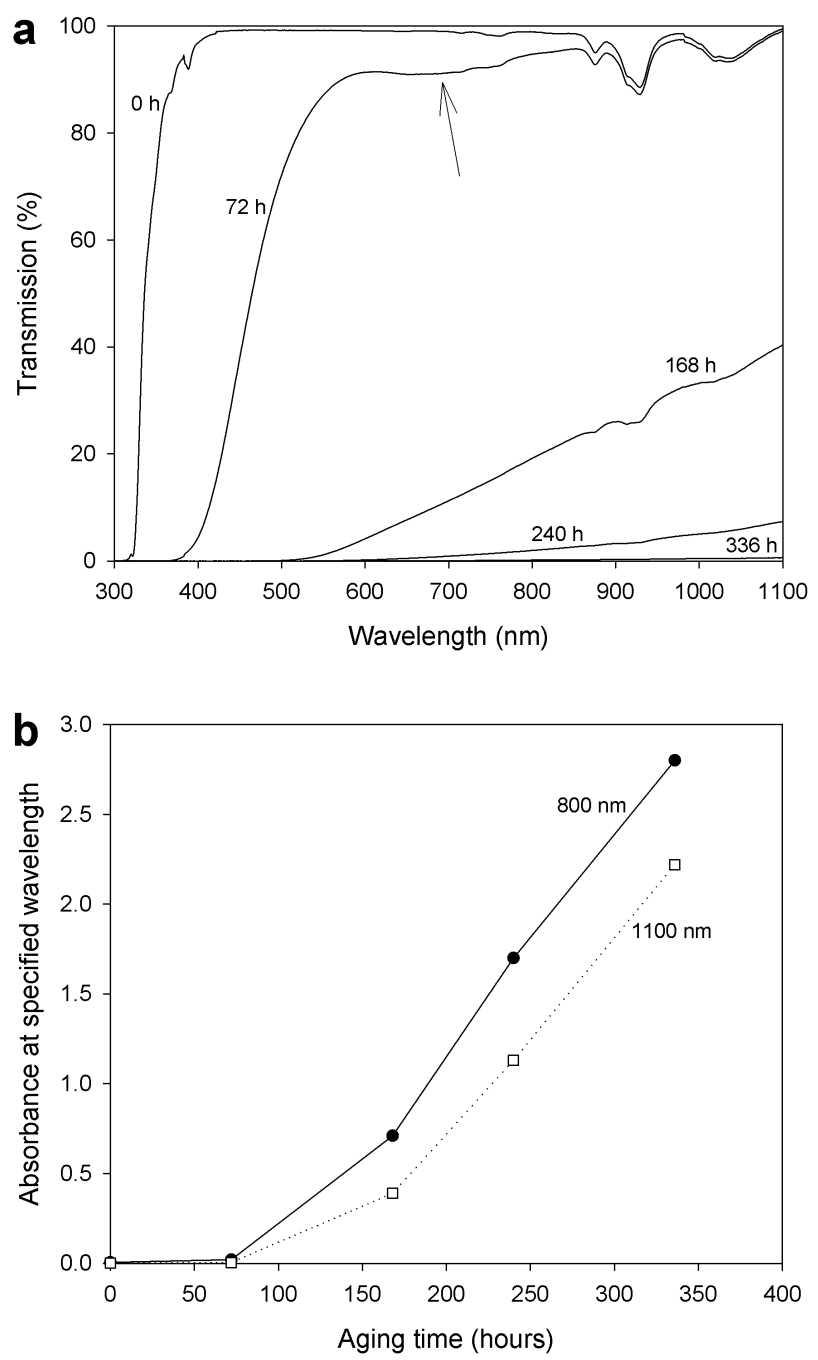

Figure 2. UV/Vis data from homogenized samples aged with copper (a) as collected spectra (b) absorbance against aging time.

\subsection{INFRARED SPECTROSCOPY}

Figure 3 shows spectra obtained from centrifuged samples (to avoid any unwanted light blocking from the precipitate).
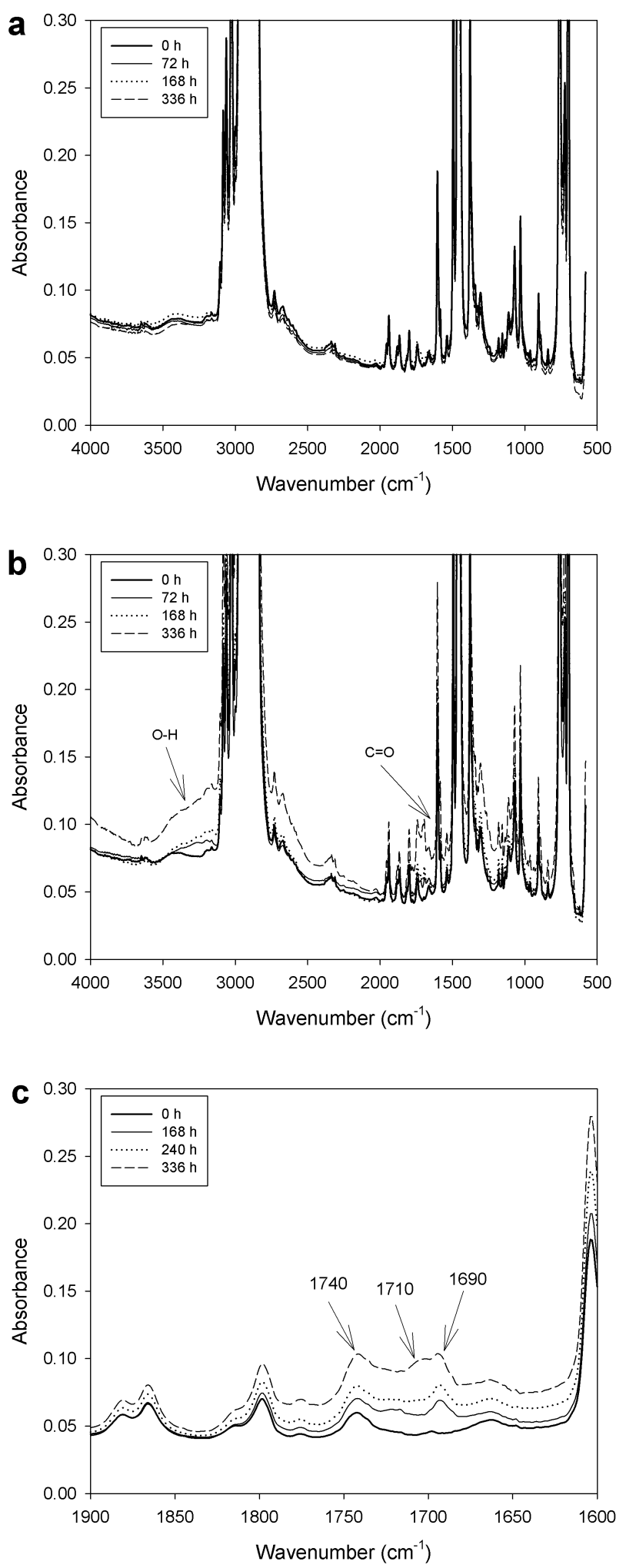

Figure 3. Infrared spectra of centrifuged oils (a) aged without copper (b) aged with copper (c) detail of carbonyl region. 
In oils aged without copper (Figure 3a) the spectra are not significantly changed after aging which indicates that negligible oxidation has occurred [23]. This should be contrasted with the previous small volume samples [18, 20, 22], which showed appreciable oxidation under the same aging conditions. A lack of oxidation in the current oils is a consequence of their much larger volume and small surface area which has restricted oxygen diffusion into the oil.

After aging with copper (Figure 3b) the spectra contain significant additional absorbance bands corresponding to the presence of hydroxyl $(\mathrm{O}-\mathrm{H})$ and carbonyl $(\mathrm{C}=\mathrm{O})$ groups indicating that oxidation is now occurring. In comparable investigations of small volume samples, only carbonyl bands were evident and it was suggested that precipitate formation serves to deplete the oil of hydroxyl containing species [18, 20]. Here the rate of precipitate production is clearly reduced resulting in a detectable quantity of hydroxyl containing molecules in solution. Therefore the observed changes in the infrared spectra in moving from small to large volume samples can be tied to restricting the level of oxygen diffusion into the oil which causes the rate of oxidation to be reduced [22].

Figure $3 \mathrm{c}$ shows the carbonyl region from samples aged with copper. Here three peaks of roughly equal magnitude were observed; $1740 \mathrm{~cm}^{-1}$ (pendent $\mathrm{C}=\mathrm{O}$ groups; i.e. esters or aldehydes) $1710 \mathrm{~cm}^{-1}$ (carboxylic acid) and $1690 \mathrm{~cm}^{-1}$ (aromatic ketones) [21]. The same three peaks, albeit at much higher intensity, were observed in identically aged small volume samples [20], except the peak at $1710 \mathrm{~cm}^{-1}$ was significantly stronger relative to the other 2 peaks. The current samples lack any obvious hydroxyl peak at $\sim 3400 \mathrm{~cm}^{-1}$ which was previously ascribed to hydroxyl groups from carboxylic acid groups. Whilst here the same compounds are formed during aging, production of carboxylic acids is clearly reduced relative to the previous small volume samples.

\subsection{WATER CONTENT}

Figure 4 shows the water content measured for all the aged oil samples (uncertainty \pm 10 PPM). The water content in the

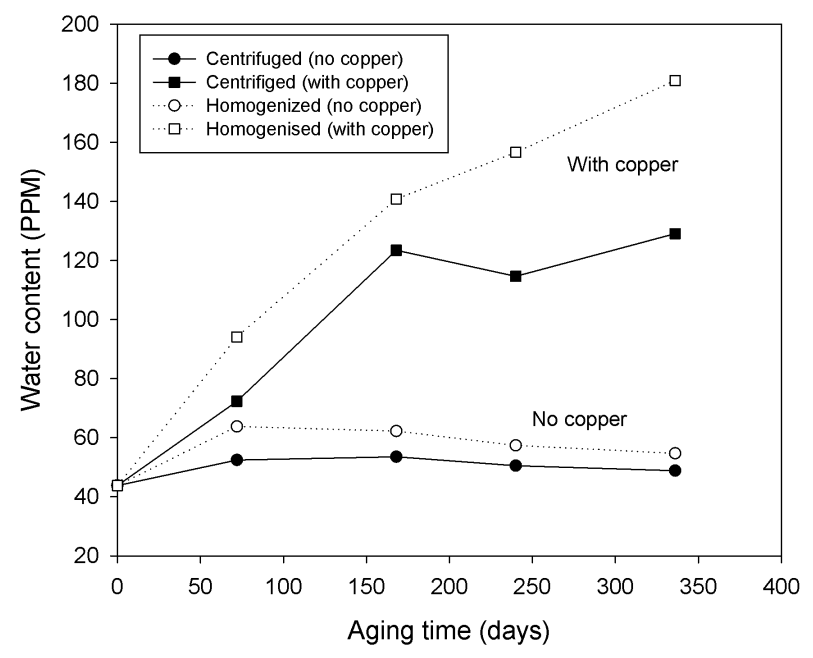

Figure 4. Water content measurements as a function of aging time. oil samples aged without copper hardly increases at all, which correlates with the lack of oxidation detected by infrared spectroscopy [22]. After aging with copper (where oxidation occurs) the water content increases to 120 PPM in centrifuged samples (closed symbols) and reaches a stable value after $168 \mathrm{~h}$ aging time; at this point the production of aging products in the oil is clearly balanced by their removal as precipitate. In homogenized samples (open symbols) the water content continues to increase (to $\sim 160$ PPM after $336 \mathrm{~h}$ ), reflecting an increasing concentration of precipitate (see Figure 2). In previous studies on homogenized small volume samples [20, 21], the water content increased to 300 PPM which reflects the significant oxidation that occurred, whilst in samples aged under nitrogen, more modest increases were observed [22]. As expected, the current samples fall somewhere between these two extremes.

\subsection{DIELECTRIC SPECTROSCOPY}

Figure 5 shows plots of relative permittivity of homogenized oils. On aging without copper (Figure 5a) the relative permittivity is not significantly affected $(2.3 \pm 0.2)$,
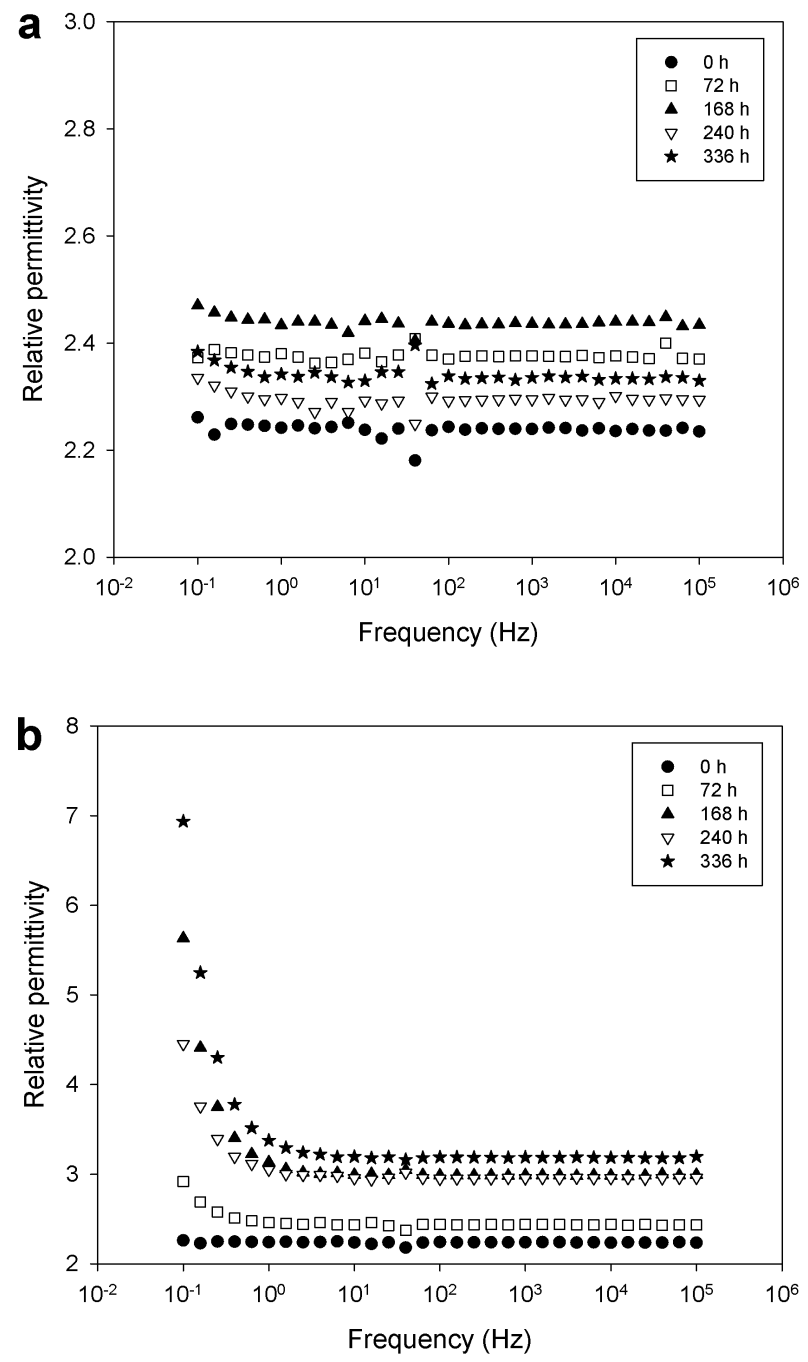

Figure 5. Relative permittivity vs frequency of homogenized oil samples aged (a) without copper (b) with copper. 
whereas aging with copper for $72 \mathrm{~h}$ results in increased values of relative permittivity (particularly at low frequencies), which then saturate after $168 \mathrm{~h}$ aging time (Figure 5b). This behavior indicates increasing dielectric polarization, hinting at increasing concentrations of polar species that are able to align in the applied field (provided that the applied frequency is not too high). As hinted at above, after $168 \mathrm{~h}$ aging time the production of aging products is balanced by their removal as precipitate, the precipitate is clearly then only having a minor effect on the dielectric properties.

Figure 6 shows the dielectric loss of the same set of homogenized oils. On aging without copper (Figure 6a) the dielectric loss at $0.1 \mathrm{~Hz}$ increases from 0.1 to 0.7 whereas that at $50 \mathrm{~Hz}$ is largely within the noise (i.e. $<3 \times 10^{-3}$ ) indicating a low loss at $\mathrm{AC}$ power frequencies but some influence of aging. On aging with copper (Figure 6b) the dielectric loss at $50 \mathrm{~Hz}$ increases from $\sim 10^{-3}$ to 0.08 with aging time, a higher loss and slope of -1 with frequency indicating an increasing level of electrical conductivity. Previous work on identically aged small volume oil samples [20] indicates that the dielectric loss
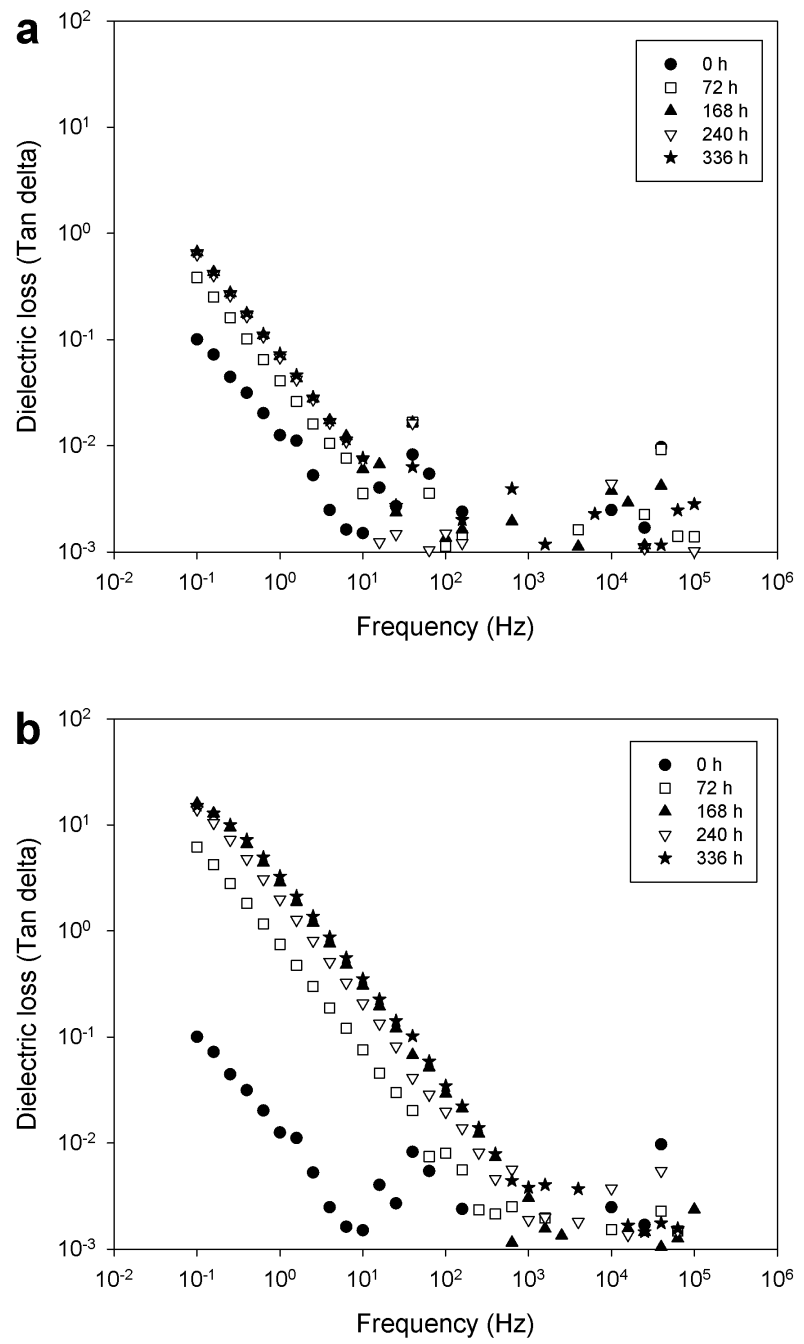

Figure 6. Dielectric loss vs frequency of homogenized oil samples aged (a) without copper (b) with copper. at $50 \mathrm{~Hz}$ increases much more after aging for $336 \mathrm{~h}$ at $135^{\circ} \mathrm{C}$; to 0.03 and 0.28 without and with copper respectively. This again confirms that the current large volume oil samples are aging far less than the previous small volume samples under the same aging conditions.

\subsection{ELECTRICAL CONDUCTIVITY}

Conductivity data are shown in Figure $7 \mathrm{a}$ for centrifuged (closed symbols) and homogenized (open symbols) samples aged without copper. Whilst the conductivity of the aged samples appears to be slightly higher than in the unaged oil, there is no overall dependence on aging time or sample treatment and the observed results all fall within the measurement uncertainties. The measured conductivity values and lack of any obvious increase in conductivity after thermal aging in the absence of copper are consistent with earlier work on silicone and vegetable oils [25, 26]. Aging with copper (Figure $7 b$ ) results in a very different behavior but again, there is no significant difference between centrifuged and homogenized oils. The conductivity is significantly increased after $72 \mathrm{~h}$ aging but clearly saturates after $168 \mathrm{~h}$, where the
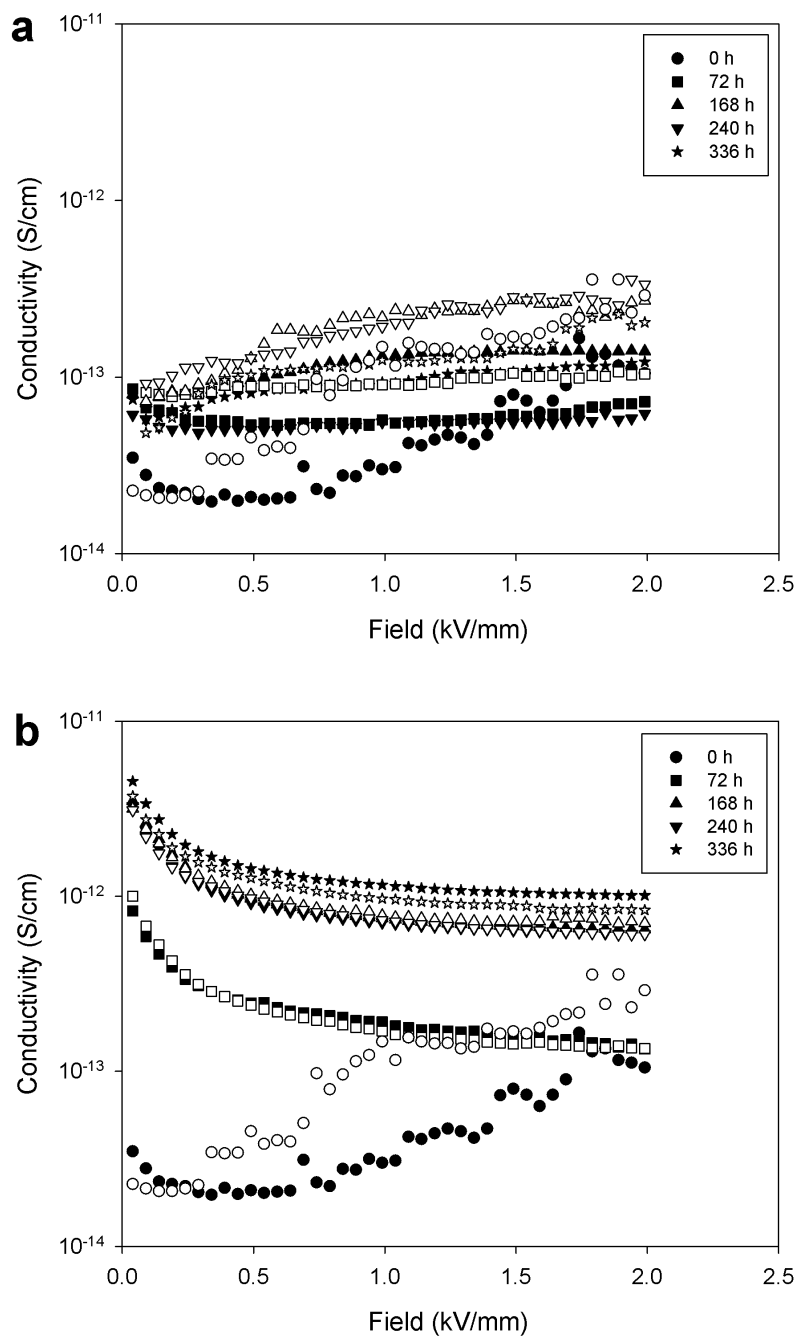

Figure 7. Electrical conductivity of samples aged (a) without copper (b) with copper. Centrifuged (closed symbols), homogenized (open symbols). 
rate of production of aging products is balanced by their removal as precipitate. The conductivity curves all show a fall with applied field a behavior that was also noted in silicone oils aged with copper [25]. This indicates that polarization dominates over any increase in conductivity due to the increase in applied field. Dielectric spectroscopy data (see Figure 5b) also suggests strong polarization in this set of oils.

\subsection{ELECTRICAL BREAKDOWN STRENGTH}

Results from AC breakdown testing are presented graphically in Figure 8 and numerical values are provided in Table 1. On aging without copper (Figure 8a), the breakdown strength falls from $14 \mathrm{kV} / \mathrm{mm}$ to $9 \mathrm{kV} / \mathrm{mm}$ after $240 \mathrm{~h}$ aging time, thus there is a deterioration in the breakdown strength of the oil after aging. The shape parameter $\beta$, which is a measure of the consistency of the measured data and is equivalent to the gradient of the fitted lines in Figure 8, is low $(\sim 5)$ indicating a large variation in the measured breakdown values.

On aging with copper, centrifuged samples (Figure 8b) show a breakdown strength that falls significantly after $72 \mathrm{~h}$ aging time indicating a more rapid deterioration in breakdown strength with aging time. The $\beta$ parameter increases to $\sim 10$ after $168 \mathrm{~h}$ aging time and remains high thereafter; the gradient of the fitted lines in Figure $8 \mathrm{~b}$ indicate far less variability in the measured breakdown values. Similar results were obtained from homogenized samples (Figure 8c) confirming that the inclusion of the precipitate does not significantly influence the electrical breakdown behavior indicating that it must be non-polar in nature. Thus it is the production of small polar molecular compounds which remain dissolved within the oil, such as water, alcohols, ketones, carboxylic acid and aldehydes [21] that cause the deterioration of the dielectric properties and not the precipitate.

The results should be contrasted with those of silicone and vegetable oils where an increase in oil viscosity led to an increase in breakdown strength [25, 26]. Here, where the viscosity is unchanged after aging [23], the breakdown strength is reduced. Similar reductions were observed in aged mineral oil [8, 9, 15], electrically aged silicone oil [24] and DDB [19]; under these conditions, electrical breakdown measurements provide a reliable diagnostic indicator of oil aging. Finally, the observed change in shape parameter $(\beta)$ may indicate a subtle change of breakdown mechanism in oils aged for long periods of time in the presence of copper. Such samples exhibited bridging [25, 27] after the breakdown event regardless of sample treatment, indicating either; (a) even after

\begin{tabular}{l|c|c|c|c}
\multicolumn{4}{c}{ Table 1. Electrical breakdown results. } \\
\hline \multirow{2}{*}{$\begin{array}{c}\text { Aging } \\
\text { conditions }\end{array}$} & \multicolumn{2}{|c|}{ Centrifuged } & \multicolumn{2}{c}{ Homogenized } \\
\cline { 2 - 5 } & $E(\mathrm{kV} / \mathrm{mm})$ & $\beta$ & $E(\mathrm{kV} / \mathrm{mm})$ & $\beta$ \\
\hline Unaged & $13.0 \pm 1.2$ & 5.4 & & \\
$72 \mathrm{~h}$ & $13.5 \pm 1.1$ & 6.9 & & \\
$168 \mathrm{~h}$ & $13.7 \pm 1.5$ & 4.9 & & \\
$240 \mathrm{~h}$ & $9.4 \pm 1.0$ & 4.5 & & \\
$336 \mathrm{~h}$ & $8.7 \pm 0.7$ & 5.6 & & 6.9 \\
$72 \mathrm{~h}+\mathrm{Cu}$ & $9.4 \pm 0.7$ & 6.1 & $9.5 \pm 0.7$ & 9.7 \\
$168 \mathrm{~h}+\mathrm{Cu}$ & $8.2 \pm 0.5$ & 8.9 & $9.2 \pm 0.5$ & 10.0 \\
$240 \mathrm{~h}+\mathrm{Cu}$ & $7.6 \pm 0.5$ & 11.8 & $9.2 \pm 0.5$ & 12.7 \\
$336 \mathrm{~h}+\mathrm{Cu}$ & $7.3 \pm 0.3$ & 13.0 & $7.7 \pm 0.4$ & \\
\hline
\end{tabular}
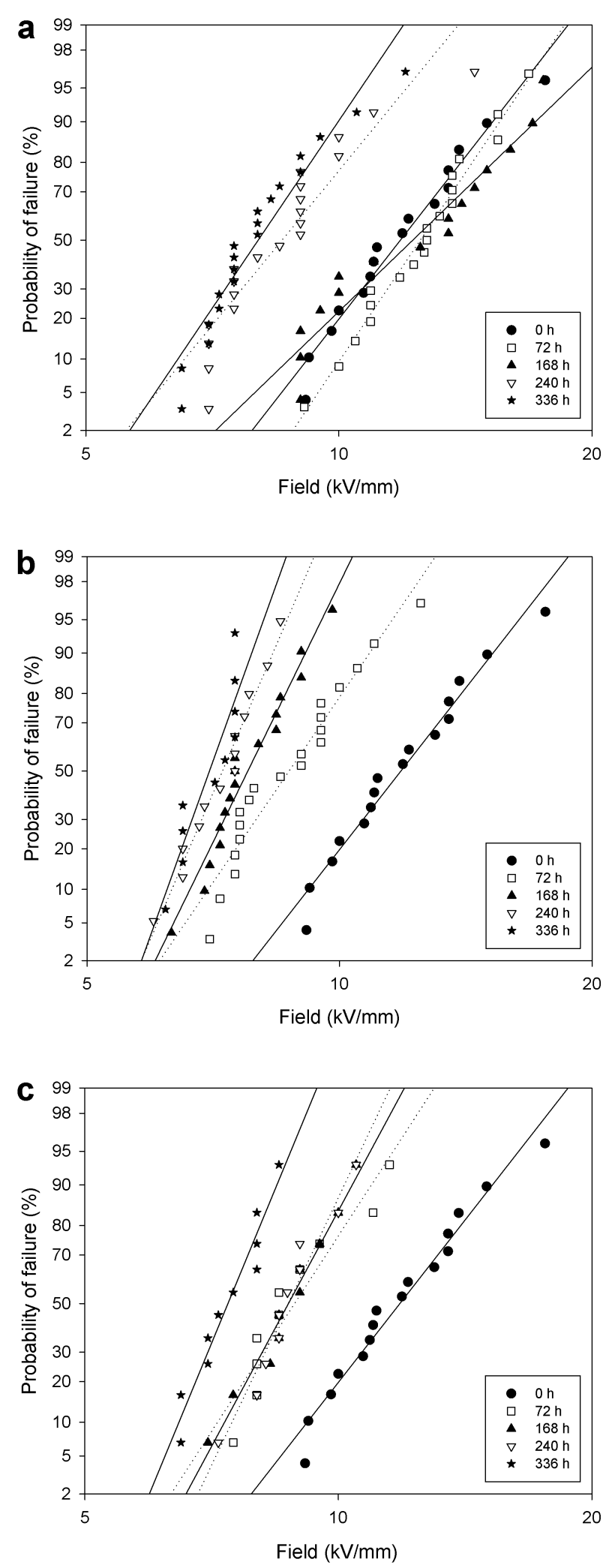

Figure 8. Weibull plots from samples aged (a) without copper) (b) centrifuged with copper (c) homogenized with copper.

centrifuging sufficient particulate matter to form a bridge remains suspended in the oil or, (b) that such particulates are formed during the breakdown event itself [19,24]. 


\section{CONCLUSIONS}

Large volume $(150 \mathrm{ml})$ samples of dodecylbenzene were thermally aged at $135^{\circ} \mathrm{C}$ in air, some samples included copper to investigate its catalytic effects. The oil yellowed and copper carboxylates were detected in samples aged with copper which eventually formed an insoluble precipitate. Compared to previous studies on much smaller volumes of oil $(5 \mathrm{ml})$, the aging rate was reduced due to a lower surface area to volume ratio and using covered containers which reduced oxygen diffusion into the oil.

Infrared spectroscopy confirmed this finding with oxidation only being detected in oils aged with copper. Absorption peaks corresponding to aldehydes, ketones and carboxylic acids were detected in agreement with earlier studies, but the level of carboxylic acid was reduced. Water content was only observed to significantly increase in samples aged with copper. In centrifuged samples the water content reached a constant value after $168 \mathrm{~h}$ aging time as the production of aging products was balanced by their removal as precipitate whilst the water content in homogenized samples continued to increase.

The relative permittivity and dielectric loss were only slightly increased in oils aged without copper but were dramatically increased when copper was included. The dielectric loss values after a prescribed period of aging were much lower than previous small volume samples confirming a much lower aging rate. Whilst aging had little effect on the dielectric properties at power frequencies, the level of polarization was dramatically increased; this could have implications if the oils were to be used in HVDC cables.

Electrical conductivity was hardly increased in the case of oils aged in the absence of copper but when aged with copper, was dramatically increased. The field dependence confirms polarization effects are dominant over any effects due to increasing applied field. The AC breakdown strength was observed to fall after aging, much more so when aging was done in the presence of copper. The precipitate formed on aging was found to have no significant effect on the electrical conductivity or electrical breakdown strength indicating that the polar products remaining in solution are responsible for the observed deterioration in dielectric properties after aging.

To confirm the role of particulates on electrical breakdown strength, additional work where known concentrations of conducting and non-conducting particulates are added to oil samples is currently underway.

\section{REFERENCES}

[1] T. K. Saha, "Review of modern diagnostic techniques for assessing insulation condition in aged transformers", IEEE Trans. Diel. Electr. Insul., Vol. 10, No. 5, pp. 903-917, 2003.

[2] M. Wang, A. J. Vandermaar and K. D. Srivastava, "Review of condition assessment of power transformers in service", IEEE Electr. Insul. Mag., Vol. 18, No. 6, pp. 12-25, 2002.

[3] C. P. McShane, "Relative properties of the new combustion resistant vegetable oil based dielectric coolants for distribution and power transformers", IEEE Trans. Ind. Appl., Vol. 37, No. 4, pp. 1132-1139, 2001 .
[4] C. P. McShane, "Vegetable oil based dielectric coolants", IEEE Ind. Appl. Mag., Vol. 8, No. 3, pp. 34-41, 2002.

[5] T. V. Oommen, "Vegetable oils for liquid filled transformers", IEEE, Electr. Insul. Mag., Vol. 18, No. 1, pp. 6-11, 2002.

[6] T. K. Saha, M. Darveniza, Z. T. Yao, D. J. T. Hill and G. Yeung, "Investigating the effects of oxidation and thermal degradation on electrical and chemical properties of power transformers insulation", IEEE Trans. Power Del., Vol. 14, No. 4, pp. 1359-1367, 1999.

[7] P. M. Mitchinson, I. L. Hosier, P. L. Lewin, A. S. Vaughan, G. C. Chen and P. Jarman, "An experiment to evaluate the benefits of processing aged transformer oil”, In Conf. Rec. 2006 IEEE Int. Symp. Electr. Insul., pp. 89-92, 2006,

[8] R. Ferguson, A. Lobeiras and J. Sabau, "Suspended particles in the liquid insulation of aging power transformers", IEEE Electr. Insul. Mag., Vol. 18, No. 4, pp. 17-23, 2002.

[9] W. J. McNutt, R. L. Provost and R. J. Whearty, "Thermal life evaluation of high temperature insulation systems and hybrid insulation systems in mineral oil", IEEE Trans. Power Del., Vol. 11, No. 3, pp. 1391-1399, 1996.

[10] R. Blue, D. Uttamchandani and O. Farish, "Infrared detection of transformer insulation degradation due to accelerated thermal aging", IEEE Trans. Diel. Electr. Insul., Vol. 5, No. 2, pp. 165-168, 1998.

[11] R. C. R. Neto, D. O. Lima, T. D. S. Pinheiro, R. F. Almeida, T. N. C. Dantas, M. S. G. Dantas, M. A. S. Araujo, C. L. Cavalcante and D. C. S. Azevedo, "Thermo-oxidative stability of mineral naphthenic insulating oils: Combined effect of antioxidants and metal passivator", Ind. Eng. Chem. Res., Vol. 43, No. 23, pp. 7428-7434, 2004

[12] T. O. Rouse, "Mineral insulating oil in transformers", IEEE Electr. Insul. Mag., Vol. 14, No. 3, pp. 6-16, 1998.

[13] L. E. Lundgaard, W. Hansen, D. Linhjell and T. J. Painter, "Aging of oil-impregnated paper in power transformers", IEEE Trans. Power Del., Vol. 19, No. 1, pp. 230-239, 2004.

[14] T. B. Whitfield, J. E. Castle, C. Saracco and M. Ali, "Transport of copper over paper in high-voltage electrical insulation", Surf. Int. Analy., Vol. 34, No. 1, pp. 176-179, 2002.

[15] S. M. Gubanski, P. Boss, G. Csepes, V. D. Houhanessian, J. Filippini, P. Guuinic, U. Gafvert, V. Karius, J. Lapworth, G. Urbani, P. Werelius and W. Zaengl, "Dielectric response methods for diagnostics of power transformers", IEEE Electr. Insul. Mag., Vol.19, No. 3, pp. 12-18, 2003.

[16] S. Velusamy and T. Punniyamurthy, "Copper(II) catalysed C-H oxidation of alkylbenzenes and cyclohexanes with hydrogen peroxide", Tetr. Letts., Vol. 44, No. 50, pp. 8955-8957, 2003.

[17] F. Behar, F. Lorant, H. Budinski and E. Desavis, "Thermal stability of alkylaromatics in natural systems: Kinetics of thermal decomposition of Dodecylbenzene", Energy and Fuels, Vol. 16, No. 4, pp. 831-841, 2002.

[18] I. L. Hosier, A. S. Vaughan, S. J. Sutton, J. Cooper, "An aging study of blends of dodecylbenzene and mineral oil", IEEE Trans. Diel. Electr. Insul., Vol. 16, No. 6, pp. 1664-1675, 2009.

[19] R. Huynh, F. J. Davis, D. Patel and A. S. Vaughan, "Degradation of Dodecylbenzene under conditions of high electric field", Diel. Mat., Meas. and Appl., IEE Conf. Publ. 473, pp 224-229, 2000.

[20] I. L. Hosier, A. S. Vaughan, S. J. Sutton and F. J. Davis, "Chemical, physical and electrical properties of aged dodecylbenzene 1: Thermal aging of mixed isomers in air", IEEE Trans. Diel. Electr. Insul., Vol. 14, No. 5, pp. 1113-1124, 2007.

[21] I. L. Hosier, A. S. Vaughan, S. J. Sutton and F. J. Davis, "Chemical, physical and electrical properties of aged Dodecylbenzene 2: Thermal aging of single isomers in air", IEEE Trans. Diel. Electr. Insul., Vol. 15, No. 5, pp. 1393-1405, 2008.

[22] I. L. Hosier, A. S. Vaughan, S. J. Sutton, J. Cooper, F. J. Davis, "Chemical, physical and electrical properties of aged dodecylbenzene 3 : Thermal aging of mixed isomers in nitrogen and under sealed conditions", IEEE Trans. Diel. Electr. Insul., Vol. 15, No. 4, pp. 10561064, 2008.

[23] I. L. Hosier, A. Guushaa, E. W. Westenbrink, C. Roger, A. S. Vaughan and S. G. Swingler, "Aging of biodegradable oils and assessment of their suitability for high voltage applications", IEEE Trans. Diel. Electr. Insul., Vol. 18, No. 3, pp. 728-738, 2011.

[24] I. L. Hosier, A. S. Vaughan, S. G. Swingler, G. Moss, "Thermal and electrical ageing of silicone oil", In Proc. 16th Int. Symp. High Volt. Eng., Paper C-8, 2009.

[25] I. L. Hosier, H. Ma, A. S. Vaughan, "Effect of electrical and thermal aging on the breakdown strength of silicone oil", In Proc. 18th IEEE Int. Conf. Diel. Liq., Paper 160, 2014 
[26] I. L. Hosier, J. Gu, W. Chotchuangchutchavel, A. S. Vaughan, "Effect of viscosity and water content on the breakdown strength of vegetable oils", In Proc. 18th IEEE Int. Conf. Diel. Liq., Paper 162, 2014.

[27] S. Mahmud, G. Chen, I. O. Golosnoy, G. Wilson, P. Jarman, "Experimental Studies of influence of DC and AC Electric Fields on Bridging in Contaminated Transformer Oil," IEEE Trans. Dielectr. Electr. Insul., Vol. 22, No. 1, pp. 152 - 160, 2015.

Preliminary results from this research project were presented at the 2016 International Conference on Dielectrics, Montpellier, France. All data published in this paper are openly available from the University of Southampton repository at http://dx.doi.org/10.5258/SOTON/397556

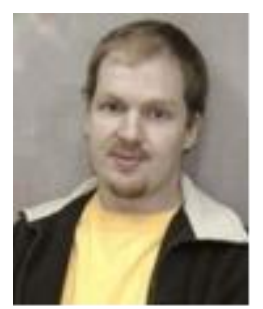

Ian Hosier was born in the UK in 1970. He received the B.Sc. degree in physics and the Ph.D. degree in polymer physics from The University of Reading, UK. He then went on with postdoctoral research at the same university, which involved extensive collaborations with institutions in Germany and Japan. In 2001 he accepted a placement at Florida State University in the USA looking at atomic force microscopy of semi-crystalline polymer blends. In 2003 he returned to the UK to take a research post at the University of Southampton looking at the aging behavior of a variety of solid and liquid insulation materials. Research interests include dielectrics, aging, microscopy and polymer morphology.

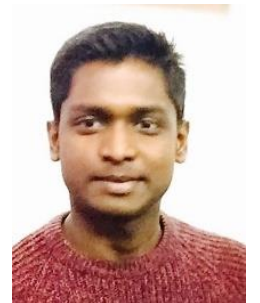

Jim Koilraj was born in India in 1993. He completed his Bachelor's degree in electrical and electronics engineering from Vi Institute of Technology, India. $\mathrm{He}$ followed it with an MSc in Energy and Sustainability with Electrical Power Engineering from the University of Southampton, UK. Through his M.Sc project Jim studied the aging behavior of Dodecybenzene before his graduation in 2015 .

Alun Vaughan (SM'07) has a B.Sc. degree in chemical physics and a Ph.D. degree in polymer physics. After working at the UK's Central Electricity

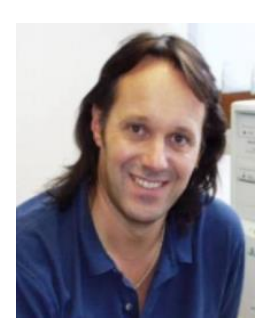
Research Laboratories and spending a period as an academic at The University of Reading, he is now Professor of Dielectric Materials and Head of the Electronics and Electrical Engineering research group at the University of Southampton. He is a former chair of The Dielectrics Group of the Institute of Physics, a Fellow of the Institute of Physics and a Fellow of the IET. 